\title{
Brain iron deposition analysis using susceptibility weighted imaging and its association with body iron level in patients with mild cognitive impairment
}

\author{
LILI GAO $^{1}$, ZHIHONG JIANG ${ }^{1}$, ZHAOCHENG CAI ${ }^{2}$, MING CAI ${ }^{1}$, QING ZHANG ${ }^{2}$, \\ YINGYING MA ${ }^{1}$, GUOLIANG LI ${ }^{1}$, FENGZHU ZHAO ${ }^{1}$ and QIANG MA $^{1}$ \\ Departments of ${ }^{1}$ Neurology and ${ }^{2}$ Radiology, Affiliated Zhongshan Hospital of \\ Dalian University, Dalian, Liaoning 116000, P.R. China
}

Received March 15, 2017; Accepted September 21, 2017

DOI: $10.3892 / \mathrm{mmr} .2017 .7668$

\begin{abstract}
The aim of the present study was to analyze brain and body iron levels among patients with mild cognitive impairment (MCI), Alzheimer's disease (AD), and normal controls (NCs). A total of 90 participants (30 MCI, $30 \mathrm{AD}, 30 \mathrm{NC}$ ) were enrolled. Brain iron content was quantified using susceptibility weighted imaging (SWI). Phase values were used to calculate bilateral iron content in the hippocampus (HP), substantia nigra, red nucleus (RN), dentate nucleus (DN), caudate nucleus $(\mathrm{CN})$, globus pallidus (GP), putamen (PUT), frontal white matter, temporal cortex (TC), and parietal cortex. Body iron indices, including serum iron, serum transferrin, ferritin and total iron binding capacity, were measured. Phase values of the left (L)-DN, L-CN, and bilateral PUT in the MCI group were significantly lower compared with $\mathrm{NC}$ group. Phase values of the right (R)-RN, bilateral DN, and bilateral PUT in the AD group were significantly lower compared with the MCI group. Phase values of the bilateral HP, DN, RN, CN, GP, PUT, and $\mathrm{L}-\mathrm{TC}$ in the $\mathrm{AD}$ group were significantly lower compared with the NC group. Serum ferritin levels in the MCI and AD groups were significantly lower compared with the NC group. Only serum iron in the AD group was positively associated with iron content in the R-HP. Brain iron deposition and body iron levels both increased in $\mathrm{MCI}$ and $\mathrm{AD}$ patients, however, excessive brain iron accumulation may have no association with body iron level. SWI was particularly effective at recognizing the presence of brain iron in the $\mathrm{MCI}$ and $\mathrm{AD}$ groups.
\end{abstract}

Correspondence to: Professor Qiang Ma, Department of Neurology, Affiliated Zhongshan Hospital of Dalian University, 6 Jiefang Street, Zhongshan, Dalian, Liaoning 116000, P.R. China E-mail: maqiangcn@126.com

Key words: mild cognitive impairment, susceptibility weighted imaging, brain iron deposition, body iron indices

\section{Introduction}

In 2011, the National Institute on Aging and the Alzheimer's Association (NIA-AA) workgroups divided Alzheimer's disease (AD) into three phases: i) Preclinical stages of AD (1), ii) mild cognitive impairment (MCI) (2), and iii) dementia due to $\mathrm{AD}$ (3). MCI is the symptomatic predementia phase of dementia, which presents clinically with mild cognition decline, but activities of daily living have not been significantly affected (4). An epidemiologic survey shows that $\sim 12-15 \%$ of cases evolve to dementia each year, and $2 / 3$ of $\mathrm{AD}$ originate from MCI (5). The existing diagnosis of MCI relies mainly on subjective symptoms and neuropsychological scales, which lack objective clinical biomarkers. Therefore, exploring objective biomarkers and developing effective treatment for $\mathrm{MCI}$ are the key to prevention of AD.

Cognitive impairment is closely related to excessive iron accumulation in the brain. As early as 1953, some pathologists found that brain iron content in senile plaques (SPs) of AD patients increased comparatively $(6,7)$. However, due to potential problems with patient compliance, no pathological studies have estimated the concentration of brain iron deposition in MCI patients. Therefore, a non-invasive and effective test for the detection of brain iron content is an urgent requirement. Susceptibility weighted imaging (SWI) is a new magnetic resonance imaging (MRI) technique based on T2WI, which is superior to conventional MRI in its ability to demonstrate paramagnetic signals. It can quantitatively analyze iron content indirectly by measuring the magnitude and phase data of this element $(8,9)$. This study aimed to investigate the concentration of brain iron deposition in MCI patients, provide objective biomarkers for early detection of dementia, especially $\mathrm{AD}$, and ultimately, aid the field in moving toward interventions at earlier stages of $\mathrm{AD}$ when some disease modifying therapies may be most efficacious.

Some studies have reported that patients with MCI and AD have body iron metabolism disorder (10), but others have considered this a highly debatable point. The relationship between brain iron deposition and body iron levels, or if the brain iron comes from body iron, remains unclear. In this study, we detected multiple serum iron indices of the study participants to identify whether patients with MCI or 
AD have abnormal body iron stores (low or high), to look for a new serum biomarker, and to simplify the early diagnosis of MCI. We also performed regression analyses on the brain iron content measured using SWI and explored the relationship between body iron homeostasis and brain iron content in patients with MCI and AD.

\section{Materials and methods}

Participants. Ninety participants [30 MCI, 30 AD, and 30 normal control (NC)] were admitted at the Affiliated Zhongshan Hospital of Dalian University from October 2015 to November 2016. The MCI group inclusion criteria were in accordance with those set by Petersen et al (11) and were as follows: i) Complaint of defective memory, ii) normal activities of daily living, iii) normal general cognitive function, iv) abnormal memory function for age, and v) absence of dementia, with scores of 19-30 on the Mini-Mental State Examination (MMSE) and 0.5 on clinical dementia rating (CDR). The $\mathrm{AD}$ group inclusion criteria were as follows: i) Met the Diagnostic and Statistical Manual of Disorders, 4th edition (DSM-IV) guidelines for AD; ii) met the National Institute of Neurological and Communicative Disorders and Stroke-Alzheimer's Diseases and Related Disorders Associations (NINCDS-ADRDA) guidelines for probable AD (12); iii) MMSE score is abnormal, illiterate group $\leq 19$ points, primary school group $\leq 22$ points, middle school and above group $\leq 24$ points, and CDR score $\geq 1$; and iv) Hachinski ischemic scale (HIS) score $<4$ points, except for vascular dementia and mixed dementia. The control group inclusion criteria were as follows: i) Age, sex, and educational level matched with patient groups; and ii) no cognitive impairment, which was determined with an MMSE score $\geq 28$ points. Exclusion criteria included structural abnormalities that could produce dementia, such as cerebrovascular disease, tumors, brain trauma, epilepsy, alcoholism, psychiatric illness, or other systemic diseases that affect brain function. This study was conducted in accordance with the declaration of Helsinki. This study was conducted with approval from the Ethics Committee of Affiliated Zhongshan Hospital of Dalian University (MEC-ZSYY-2015-10). Written informed consent was obtained from all participants.

MRI procedure and data acquisition. All participants underwent clinical brain MRI on a Siemens Magnetom Verio 3.0T MR with a 12-channel phased array head coil. The conventional MRI sequences including axial T1WI, T2WI, T2WI-FLAIR, and DWI were first acquired to exclude stroke, trauma, tumor, and other intracranial lesions. SWI images were taken through axial and oblique coronal scan using three-dimensional gradient echo sequence, which is used for visualization of the HP and other regions of interest (ROIs). The image parameters for SWI were: Echo time (TE) $20 \mathrm{~ms}$, repetition time (TR) $28 \mathrm{~ms}$, slice thickness $1.8 \mathrm{~mm}$, flip angle $15^{\circ}$, average 1 , field of view (FOV) $230 \mathrm{~mm}$, voxel size (VS) $0.5 \times 0.5 \times 1.2 \mathrm{~mm}$. The original images taken using SWI were processed using the post-processing software of the Siemens 3.0T MR, which generated four images: Magnitude image, filtered phase image, minimum intensity projection (mIP) image, and susceptibility-weighted image. The phase values of ROIs were measured on the filtered phase images using signal processing in MRI (SPIN) software. First, the ROIs were drawn on magnitude images according to the anatomical structures and pixel size, including bilateral hippocampus (HP), substantia nigra (SN), RN, dentate nucleus (DN), caudate nucleus $(\mathrm{CN})$, globus pallidus (GP), putamen (PUT), frontal white matter (FWM), temporal cortex (TC), and parietal cortex (PC). Through the 'copy boundary to all other views' function of the SPIN software, the boundaries of the ROIs on the magnitude images were copied to the corresponding positions in the filtered phase images. Then, the software automatically calculated the phase value of each ROI (Fig. 1). Data for each ROI were taken from the largest sections and measured three times to obtain the mean. The cerebrospinal fluid (CSF), skull, and large blood vessels were avoided when creating the outlines of the ROIs so that the accuracy is not affected. According to the formula provided by Siemens, $\varphi=2,048(\Phi+\pi) / \pi$, the phase value $(\varphi)$ was converted to the radian angle value $(\Phi)$.

We correlated the radian angle values for the bilateral $\mathrm{SN}$, red nucleus, DN, CN, GP, putamen, FWM, TC, and PC in the NC group with the postmortem brain non-hemoglobin iron content reported by Hallgren and Sourander (13) to verify our data. We also calculated a formula between radian angle values and brain iron content to depict their relationship. Using this formula, the phase value was converted to the brain iron content of the corresponding region, which could be used for the following data analysis.

Serum iron indices evaluation. After a 12-h fast, $5 \mathrm{ml}$ venous blood samples were collected and centrifuged within $10 \mathrm{~min}$ at 3,000 rpm. The serum supernatant was stored at $-80^{\circ} \mathrm{C}$ for the measurement of serum iron parameters. Serum iron was evaluated using direct colorimetric methods (Meikang Chemical Industries, Ningbo, China). Serum transferrin levels were quantitatively detected using an immunoturbidimetric assay (Siemens Healthcare Diagnostics Products Gmb, Marburg, Germany). Serum ferritin was determined using Chemiflex (Abbott Ireland Diagnostic Dvision, Longford, Ireland) and total iron binding capacity was measured using ADVIA Chemistry XPT (Siemens Healthcare Diagnostics Inc., Deerfield, IL, USA). All levels were quantified following the manufacturer's instructions to avoid artificial errors.

Statistical analysis. For statistical analysis, SPSS 17.0 was used. Data were presented as mean value \pm standard deviation (SD). Categorical variables were analyzed using the Chi-square test. One-way analysis of variance (ANOVA) model was used to compare values among three groups using Fisher's least significant difference (LSD) test (two sample t-test comparison). Pearson correlation coefficient was used to analyze the relationship between quantitative variables. Because of the large variance of serum ferritin, we used the logarithm (base 10) of serum ferritin instead in the correlation analysis. P-value $<0.05$ was considered to indicate a statistically significant difference.

\section{Results}

Baseline characteristics of participants. The demographics and neuropsychological data of participants are summarized 


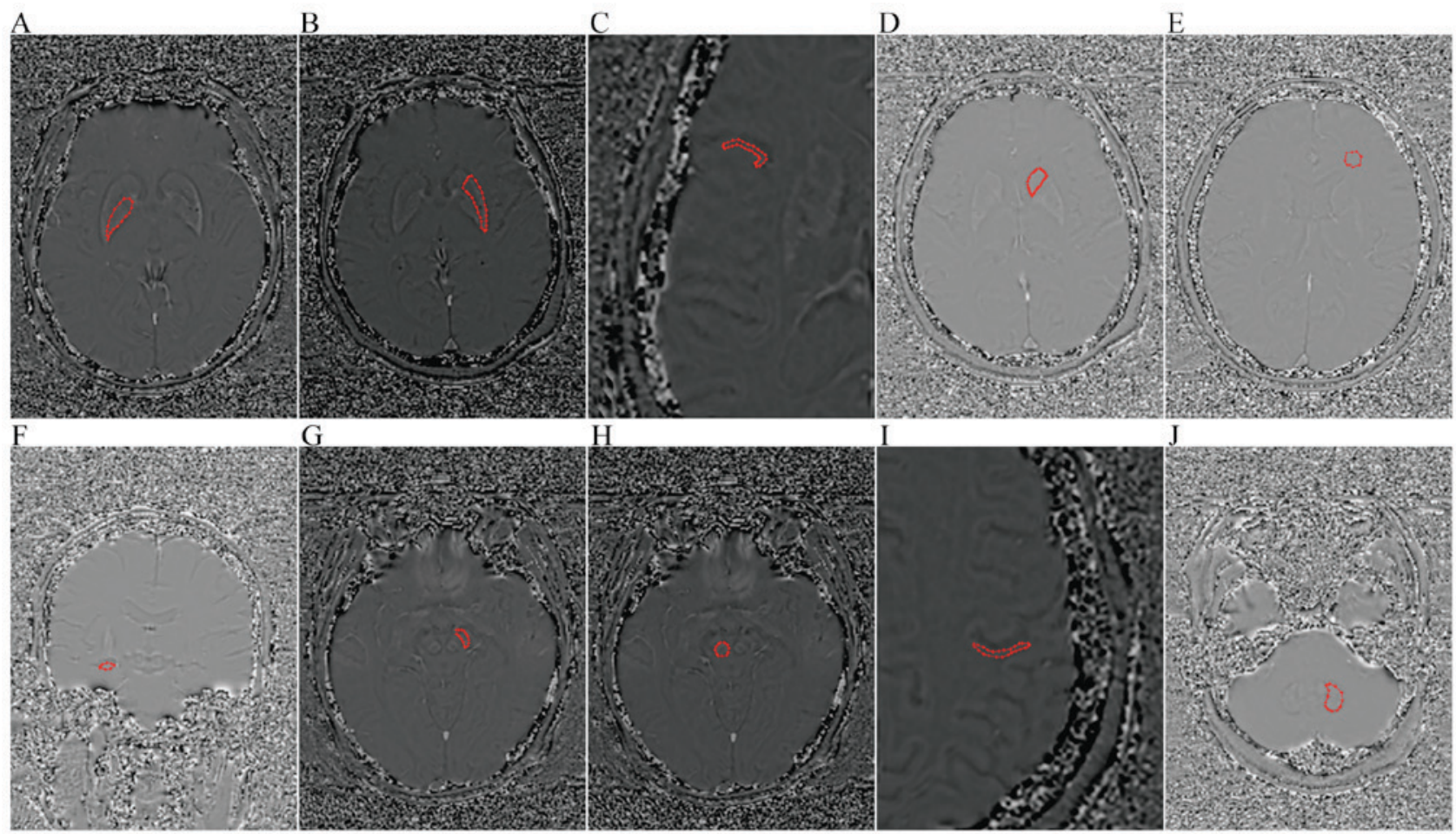

Figure 1. Axial and coronal filtered phase image showing the ROIs. (A) GP, (B) PUT, (C) TC, (D) CN, (E) FWM, (F) HP, (G) SN, (H) RN, (I) PC, and (J) DN ROIs, regions of interest; GP, globus pallidus; PUT, putamen; TC, temporal cortex; CN, caudate nucleus; FWM, frontal white matter; HP, hippocampus; $\mathrm{SN}$, substantia nigra; RN, red nucleus; PC, parietal cortex; DN, dentate nucleus.

Table I. Demographics and neuropsychological data of participants.

\begin{tabular}{lccccr}
\hline Characteristics & AD & MCI & NC & F-value & P-value \\
\hline Number & 30 & 30 & 30 & & \\
Sex (female/male) & $18 / 12$ & $13 / 17$ & $17 / 13$ & 1.87 & 0.39 \\
Age (years) & $74.83 \pm 4.52$ & $75.2 \pm 5.75$ & $72.86 \pm 5.75$ & 1.74 & 0.18 \\
MMSE & $17.76 \pm 4.15$ & $27.13 \pm 1.19$ & $28.73 \pm 1.11$ & 158.27 & $<0.05^{\mathrm{a}}$ \\
MoCA & $10.86 \pm 3.62$ & $20.13 \pm 3.02$ & $25.40 \pm 2.01$ & 184.91 & $<0.05^{\mathrm{a}}$ \\
\hline
\end{tabular}

${ }^{\mathrm{a}} \mathrm{P}<0.05$, indicating a significant difference between the two groups. $\mathrm{AD}$, Alzheimer's disease; MCI, mild cognitive impairment; NC, normal control; MMSE, Mini-Mental State Examination; MoCA; Montreal Cognitive Assessment.

in Table I. Sex and age among the three groups are matched. The MMSE and Montreal Cognitive Assessment (MoCA) scores of the $\mathrm{AD}, \mathrm{MCI}$, and $\mathrm{NC}$ groups increased gradually, and the difference was statistically significant.

Difference of phase values of ROIs among three groups. The radian angle values of the ROIs are shown in Table II and Fig. 2. Brain iron deposition compared among the MCI, AD, and NC groups were as follows: The radian angle values of the left DN, left $\mathrm{CN}$, and bilateral PUT of the MCI group were significantly lower than those of the $\mathrm{NC}$ group. The radian angle values of the bilateral DN, right red nucleus, and bilateral PUT of the AD group were significantly lower than those of the MCI group. The radian angle values of the bilateral HP, bilateral $\mathrm{DN}$, bilateral red nucleus, bilateral $\mathrm{CN}$, bilateral GP, bilateral putamen, and left $\mathrm{TC}$ in the $\mathrm{AD}$ group were significantly lower than those in the $\mathrm{NC}$ group.
Correlations between phase values and brain iron concentrations of $N C$. There is a significantly negative correlation between the phase values of ROIs and the postmortem brain non-hemoglobin iron content reported by Hallgren and Sourander (13) $(R=-0.9391, \mathrm{P}<0.05$; Fig. 3A). The linear formula is: $y=-0.0063 x+0.035$, where $y$ is the phase value, and $\mathrm{x}$ is the brain iron concentration.

Difference of body iron indices among the three groups. Table III presents the body iron indices of the three groups. Compared with the NC group, serum ferritin in MCI group and $\mathrm{AD}$ group were significantly higher, suggesting that the patients with $\mathrm{MCI}$ and $\mathrm{AD}$ may have body iron overload.

Correlation between body iron indices with regional brain iron concentration in the MCI and AD groups. Theregression analyses show that only serum iron in AD group is positively correlated 
Table II. Radian angle value of the ROIs in AD, MCI and NC groups.

\begin{tabular}{|c|c|c|c|c|c|c|}
\hline \multirow[b]{2}{*}{ ROIs } & \multirow[b]{2}{*}{$\mathrm{NC}$} & \multirow[b]{2}{*}{ MCI } & \multirow[b]{2}{*}{$\mathrm{AD}$} & \multicolumn{3}{|c|}{ Comparison between group (P) } \\
\hline & & & & 12 & 23 & 13 \\
\hline \multirow[t]{2}{*}{ HP } & R: $0.0372 \pm 0.0141$ & $0.0348 \pm 0.0126$ & $0.0301 \pm 0.0084$ & 0.429 & 0.136 & $0.024^{\mathrm{a}}$ \\
\hline & L: $0.0366 \pm 0.0119$ & $0.0321 \pm 0.0099$ & $0.0294 \pm 0.0097$ & 0.106 & 0.320 & $0.010^{\mathrm{a}}$ \\
\hline \multirow[t]{2}{*}{$\mathrm{DN}$} & $\mathrm{R}:-0.0456 \pm 0.0073$ & $-0.0497 \pm 0.0064$ & $-0.0876 \pm 0.0103$ & 0.057 & $<0.05^{\mathrm{a}}$ & $<0.05^{\mathrm{a}}$ \\
\hline & L: $-0.0515 \pm 0.0062$ & $-0.0604 \pm 0.0092$ & $-0.0933 \pm 0.0088$ & $<0.05^{\mathrm{a}}$ & $<0.05^{\mathrm{a}}$ & $<0.05^{\mathrm{a}}$ \\
\hline \multirow[t]{2}{*}{$\mathrm{RN}$} & R: $-0.0816 \pm 0.0053$ & $-0.0832 \pm 0.0051$ & $-0.0888 \pm 0.0063$ & 0.272 & $<0.05^{\mathrm{a}}$ & $<0.05^{\mathrm{a}}$ \\
\hline & L: $-0.0868 \pm 0.0049$ & $-0.0889 \pm 0.0058$ & $-0.0907 \pm 0.0068$ & 0.169 & 0.241 & $0.012^{a}$ \\
\hline \multirow[t]{2}{*}{$\mathrm{SN}$} & R: $-0.0826 \pm 0.0088$ & $-0.0846 \pm 0.0062$ & $-0.0853 \pm 0.0073$ & 0.305 & 0.694 & 0.157 \\
\hline & L: $-0.0785 \pm 0.0087$ & $-0.0803 \pm 0.006$ & $-0.0810 \pm 0.0054$ & 0.313 & 0.712 & 0.169 \\
\hline \multirow[t]{2}{*}{$\mathrm{CN}$} & R: $-0.0459 \pm 0.0091$ & $-0.0488 \pm 0.0061$ & $-0.0501 \pm 0.0058$ & 0.124 & 0.492 & $0.027^{\mathrm{a}}$ \\
\hline & L: $-0.0591 \pm 0.0084$ & $-0.0645 \pm 0.0107$ & $-0.0656 \pm 0.0097$ & $0.032^{\mathrm{a}}$ & 0.672 & $0.011^{\mathrm{a}}$ \\
\hline \multirow[t]{2}{*}{ GP } & R: $-0.0817 \pm 0.0119$ & $-0.0832 \pm 0.0095$ & $-0.0877 \pm 0.0099$ & 0.578 & 0.100 & $0.029^{\mathrm{a}}$ \\
\hline & L: $-0.0881 \pm 0.0097$ & $-0.0922 \pm 0.0097$ & $-0.0941 \pm 0.0110$ & 0.115 & 0.464 & $0.022^{\mathrm{a}}$ \\
\hline \multirow[t]{2}{*}{ PUT } & R: $-0.0426 \pm 0.0079$ & $-0.0479 \pm 0.006$ & $-0.0555 \pm 0.0056$ & $0.003^{\mathrm{a}}$ & $<0.05^{\mathrm{a}}$ & $<0.05^{\mathrm{a}}$ \\
\hline & L: $-0.0499 \pm 0.0077$ & $-0.0548 \pm 0.0066$ & $-0.0626 \pm 0.0058$ & $0.006^{\mathrm{a}}$ & $<0.05^{\mathrm{a}}$ & $<0.05^{\mathrm{a}}$ \\
\hline \multirow[t]{2}{*}{ FWM } & R: $0.0029 \pm 0.0077$ & $0.0005 \pm 0.0094$ & $-0.0005 \pm 0.0062$ & 0.242 & 0.618 & 0.097 \\
\hline & L: $0.0054 \pm 0.0079$ & $0.0017 \pm 0.0116$ & $0.0015 \pm 0.0076$ & 0.122 & 0.940 & 0.106 \\
\hline \multirow[t]{2}{*}{ TC } & R: $0.0294 \pm 0.0094$ & $0.0271 \pm 0.0079$ & $0.0265 \pm 0.0071$ & 0.285 & 0.772 & 0.176 \\
\hline & L: $0.0242 \pm 0.0075$ & $0.0210 \pm 0.0058$ & $0.0188 \pm 0.0077$ & 0.087 & 0.232 & $0.004^{\mathrm{a}}$ \\
\hline \multirow[t]{2}{*}{$\mathrm{PC}$} & R: $0.0379 \pm 0.0098$ & $0.0375 \pm 0.006$ & $0.0343 \pm 0.0062$ & 0.843 & 0.102 & 0.067 \\
\hline & L: $0.0306 \pm 0.0071$ & $0.0305 \pm 0.0056$ & $0.0278 \pm 0.0060$ & 0.950 & 0.097 & 0.086 \\
\hline
\end{tabular}

${ }^{\mathrm{a}} \mathrm{P}<0.05$, indicating a significant difference between the two groups; HP, hippocampus; SN, substantia nigra; RN, red nucleus; DN, dentate nucleus; CN, caudate nucleus; GP, globus pallidus; PUT, putamen; FWM, frontal white matter; TC, temporal cortex; PC, prefrontal cortex; R, right; L, left.
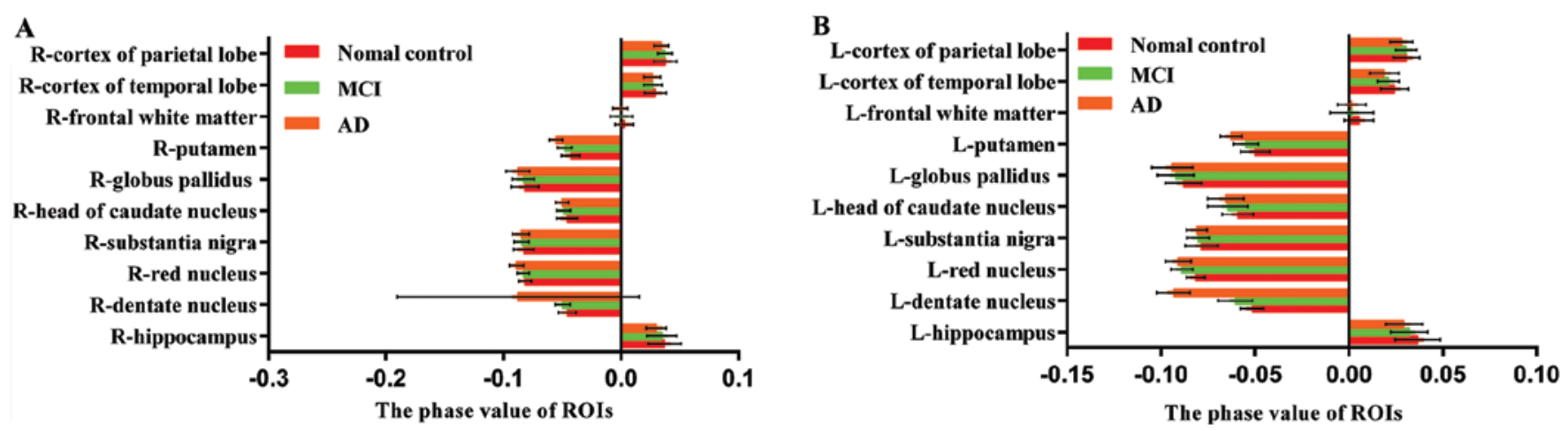

Figure 2. (A) Radian angle values of the R-ROIs in AD group, MCI group and NC group. (B) Radian angle values of the L-ROIs in AD group, MCI group and NC group. The radian angle value of L-dentate nucleus, $\mathrm{L}$-caudate nucleus and bilateral putamen of MCI group were significantly lower than those of NC group. The radian angle value of bilateral dentate nucleus, R-red nucleus and bilateral putamen of AD group were significantly lower than those of MCI group. The radian angle value of bilateral HP, bilateral dentate nucleus, bilateral red nucleus, bilateral head-caudate nucleus, bilateral globus pallidus, bilateral putamen and L-cortex of temporal lobe in $\mathrm{AD}$ group were significantly lower than those in $\mathrm{NC}$ group. $\mathrm{AD}$, Alzheimer's disease; $\mathrm{MCI}$, mild cognitive impairment; $\mathrm{NC}$, normal control; $\mathrm{HP}$, hippocampus.

with iron content in the right $\mathrm{HP}(R=0.43, \mathrm{P}=0.0402$; Fig. $3 \mathrm{~B})$. No significant correlation was found between body iron indices and iron content in the rest of the ROIs.

\section{Discussion}

An increasing number of studies are beginning to focus on a neurotoxic role for excessive iron deposition in brain, and their main findings include oxidative stress injury and lack of antioxidant system induced by the accumulation of iron (14). Wu et al (15) found in 1894 that reactive oxygen species (ROS), such as hydrogen peroxide $\left(\mathrm{H}_{2} \mathrm{O}_{2}\right)$, turns into the strongest oxidizing agent, hydroxyl radical $(\mathrm{OH})$, under the catalysis of free iron. Many studies confirmed that the brain iron content of AD patients increased, and concentrated near SPs $(16,17)$. Some scholars find that iron slows the progression of the 
Table III. Body iron indices of three groups.

P-value

\begin{tabular}{lcccccc} 
Body iron & NC & MCI & AD & NC vs. MCI & MCI vs. AD & NC vs. AD \\
\hline Serum iron $(\mu \mathrm{mol} / \mathrm{l})$ & $13.28 \pm 5.39$ & $13.97 \pm 4.23$ & $13.90 \pm 6.80$ & 0.714 & 0.970 & 0.732 \\
Serum ferritin $(\mathrm{ng} / \mathrm{ml})$ & $89.18 \pm 37.45$ & $172.50 \pm 115.3$ & $172.83 \pm 93.3$ & $0.017^{\mathrm{a}}$ & 1.000 & $0.001^{\mathrm{a}}$ \\
Serum transferrin $(\mathrm{mg} / \mathrm{ml})$ & $1.88 \pm 0.14$ & $1.89 \pm 0.32$ & $1.88 \pm 0.84$ & 0.944 & 0.919 & 0.980 \\
TIBC $(\mu \mathrm{mol} / \mathrm{l})$ & $47.47 \pm 6.48$ & $50.65 \pm 8.01$ & $46.51 \pm 7.34$ & 0.195 & 0.071 \\
\hline
\end{tabular}

${ }^{a} \mathrm{P}<0.05$, indicating a significant difference between the two groups; TIBC, total iron binding capacity; AD, Alzheimer's disease; MCI, mild cognitive impairment; NC, normal control.
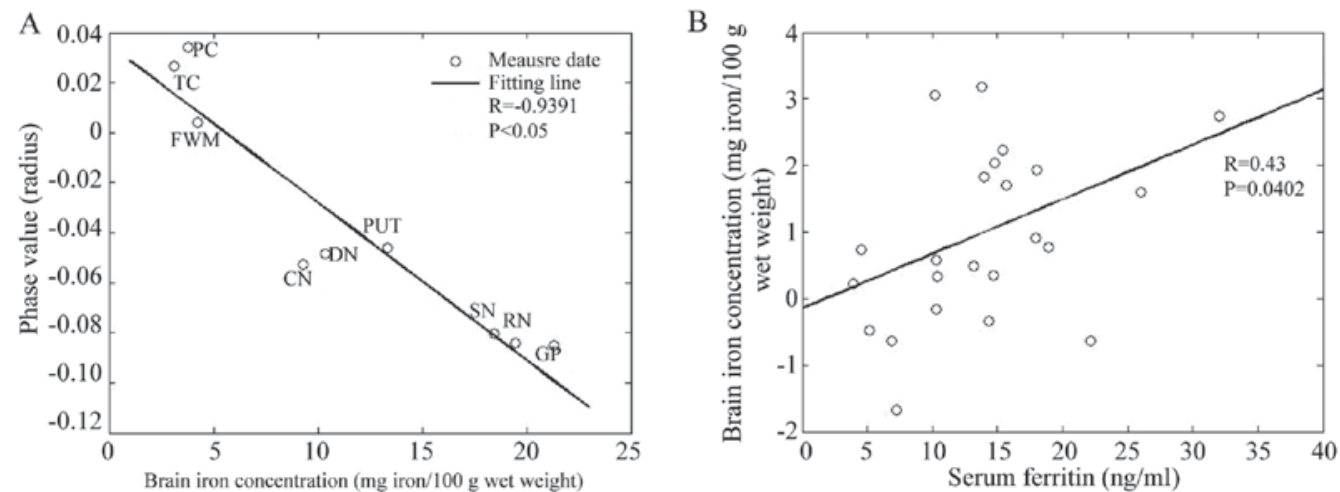

Figure 3. (A) Correlation of phase value with postmortem brain iron content in NC group. Plots of bilateral average phase values for nine brain regions in NC group. (B) Correlation of serum iron with iron of right HP in AD group. NC, normal control; HP, hippocampus; AD, Alzheimer's disease; SN, substantia nigra; RN, red nucleus; DN, dentate nucleus; CN, caudate nucleus; GP, globus pallidus; PUT, putamen; FWM, frontal white matter; TC, temporal cortex; and PC, parietal cortex.

amyloid beta peptide from an unstructured conformation to the ordered cross- $\beta$ fibrils that are characteristic of amyloid, and these data support the hypothesis that iron delays the formation of well-ordered aggregates of $\mathrm{A} \beta$ and thus, promotes its toxicity in Alzheimer disease (18). In addition, ferric iron can bind to a particular target of Tau protein in neurons and turn it into phosphorylated tau (P-tau), leading to the formation of neurofibrillary tangles (NFT) (19). Ayton et al (20) analyzed CSF levels of A $\beta 1-42$, tau, apolipoprotein E (APOE), ferritin, factor $\mathrm{H}$, and hemoglobin of participants, and the scores for cognition were measured using the longitudinal Rey Auditory-Visual Learning Task (RAVLT) and the AD Assessment Scale-cognitive subset (ADAS-Cog13). They found that ferritin levels for cognitively normal individuals were associated with cognitive deterioration. The categorization of cognitively normal individuals according to $\varepsilon 4$ revealed that the ferritin level was strongly associated with cognitive decline in $\varepsilon 4$ carriers, and the $\varepsilon 4$ allele of APOE confers the greatest genetic risk for AD. These findings demonstrate the potential for CSF ferritin as a biomarker, especially for $\varepsilon 4$ carriers, and provide new insight into the pathophysiologic mechanisms of AD. In this study, we used the SWI technique to quantitatively assess iron accumulation of ROIs in the MCI, AD, and NC groups. The results indicated that multiple brain areas of AD patients have brain iron overload, which is consistent with previous studies. In addition, we found that the iron content of the left $\mathrm{DN}$, left $\mathrm{CN}$, and bilateral PUT in patients with MCI were more than those in the NC group, suggesting that metabolic disorder of brain iron is involved in the initial stage of dementia and may be risk factors for MCI and AD. However, whether brain iron levels serve as a pathophysiological biomarker for MCI and AD still need further and repeatable histological experiments to verify.

In the brain, iron is stored in the form of ferritin, a strong paramagnetic element that aligns along the main magnetic field, which can cause a phase shift in the plaque voxel. The region of the object where there is a phase difference will then change its signal susceptibility inhomogeneities. At a given echo time, the more iron content in the tissue, the more the phase differs from zero. Therefore, any changes in the amount of iron will lead to changes in the phase of the tissue relative to its surroundings (21). There is a high correlation between phase value of SWI and the brain iron concentration. Previous studies have shown that there is a significant negative correlation between the average phase values of each ROI measured by SWI and the postmortem brain non-hemoglobin iron content in adults reported by Hallgren and Sourander, which was also validated by this study. SWI has been shown to be very sensitive to iron, offering the ability to measure it on brain tissue in vivo.

In a previous study, serum iron parameters were determined in 818 older individuals who participated in a 
3-year randomized, placebo-controlled double blind trial; cross-sectional linear regression analyses indicated that higher serum ferritin levels were significantly associated with decreased cognitive function, such as complex speed, and information-processing speed (22). However, some scholars have a different view. Milward et al (23) have examined the longitudinal relationship between serum ferritin and cognition in 800 community-dwelling Australians 60 years or older. All participants completed the Cambridge Cognitive Examination (CAMCOG) and CDR, and no relationships were observed between serum iron or transferrin saturation and the presence or absence of dementia. A meta-analysis of serum metal mineral metabolism in patients with AD also showed no change in serum iron levels in AD patients (24). The reason for this inconsistency may be because the above studies use different neuropsychological tests, and the cognitive domain emphasized in each test is not consistent. Some studies revealed that, differences in body iron levels could also be due to the different mean ages and different sex ratios. The present study observed that compared to the NC group, the serum ferritin levels of the MCI group and AD group were significantly higher. Although the total iron binding capacity and serum transferrin of MCI and AD patients were not significantly different from those in the NC group, there was a decreasing tendency. The serum iron levels showed an increasing tendency in all the three groups, indicating that MCI and AD patients maybe have systemic iron overload. The results were not statistically significant mainly because the sample size was too small; hence, we cannot completely reject the above conclusions. This conclusion was not robust and constitute an important part for study, particularly from the aspect of therapeutic interventions, such as the use of desferrioxamine in AD patients.

Iron deposition in localized brain areas increased abnormally in patients with MCI and AD. This was confirmed both by this study and a previous experiment. However, the pathological mechanisms by which brain iron accumulates are not thoroughly explained. House et al (25) compared brain R2 values with serum iron indices, and the results suggested that iron levels in specific gray matter brain regions are influenced by systemic iron status in elderly men. Some studies found that decreased serum hepcidin levels were correlated with excessive iron accumulation in the basal ganglia in patients with HBV-related cirrhosis (26). Other studies suggest that brain iron concentrations in specific regions may be related to body iron status. In this study, to investigate the possible causes of excessive brain iron deposition in the MCI and AD groups, we correlated the brain iron content of each ROI with serum iron, serum ferritin, transferrin, and total iron binding capacity. The results show that only the iron content of the right HP in the AD group was positively correlated with serum iron level, and the iron content of the rest of ROIs in the AD group and all ROIs in the MCI group have no significant correlation with body iron indices. Since only one ROI in the AD group was found to be correlated with body iron indices, even the correlation is not very significant. Whether brain iron deposition is determined by the system iron metabolism in MCI and AD patients remains unclear, and requires confirmation from further studies with large sample sizes.

\section{References}

1. Sperling RA, Aisen PS, Beckett LA, Bennett DA, Craft S, Fagan AM, Iwatsubo T, Jack CR Jr, Kaye J, Montine TJ, et al: Toward defining the reclinical stages of Alzheimer's disease: Recommendations from the National Institute on Aging-Alzhermer's Association workgroups on diagnostic guidelines for Alzheimer's disease. Alzheimers Dement 7: 280-292, 2011.

2. Albert MS, DeKosky ST, Dickson D, Dubois B, Feldman HH, Fox NC, Gamst A, Holtzman DM, Jagust WJ, Petersen RC, et al: The diagnosis of mild cognitive impairment due to Alzheimer's disease: Recommendations from the National Institute on Aging-Alzheimer's Association workgroups on diagnostic guidelines for Alzheimer's disease. Alzheimers Dement 7: 270-279, 2011.

3. McKhann GM, Knopman DS, Chertkow H, Hyman BT, Jack CR Jr, Kawas CH, Klunk WE, Koroshetz WJ, Manly JJ, Mayeux R, et al: The diagnosis of dementia due to Alzheimer's disease: Recommendations from the National Institute on Aging-Alzheimer's Association workgroups on diagnostic guidelines for Alzheimer's disease. Alzheimers Dement 7: 263-269, 2011.

4. Petersen RC: Mild cognitive impairment. Continuum (Minneap Minn) 22: 404-418. 2016.

5. Ota K, Oishi N, Ito K and Fukuyama H; SEAD-J Study Group; Alzheimer's Disease Neuroimaging Initiative: Effects of imaging modalities, brain atlases and feature selection on prediction of Alzheimer's disease. J Neurosci Methods 256: 168-183, 2015.

6. Goodman L: Alzheimer's disease; a clinico-pathologic analysis of twenty-three cases with a theory on pathogenesis. J Nerv Ment Dis 118: 97-130, 1953.

7. Honda K, Casadesus G, Petersen RB, Perry G and Smith MA: Oxidative stress and redox-active iron in Alzheimer's disease. Ann N Y Acad Sci 1012: 179-182, 2004.

8. Chamberlain R, Reyes D, Curran GL, Marjanska M, Wengenack TM, Poduslo JF, Garwood M and Jack CR Jr: Comparison of amyloid plaque contrast generated by T2-weighted, T2*-weighted, and susceptibility-weighted imaging methods in transgenic mouse models of Alzheimer's disease. Magn Reson Med 61: 1158-1164, 2009.

9. Zhou XX, Qin HL, Li XH, Huang HW, Liang YY, Liang XL and $\mathrm{Pu}$ XY: Characterizing brain mineral deposition in patients with Wilson disease using susceptibility-weighted imaging. Neurol India 62: 362-366, 2014.

10. Crespo ÂC, Silva B, Marques L, Marcelino E, Maruta C, Costa S, Timóteo A, Vilares A, Couto FS, Faustino P, et al: Genetic and biochemical markers in patients with Alzheimer's disease support a concerted systemic iron homeostasis dysregulation. Neurobiol Aging 35: 777-785, 2014.

11. Petersen RC, Smith GE, Waring SC, Ivnik RJ, Tangalos EG and Kokmen E: Mild cognitive impairment: Clinical characterization and outcome. Arch Neurol 56: 303-308, 1999.

12. Dubois B, Feldman HH, Jacova C, Dekosky ST, Barberger-Gateau P, Cummings J, Delacourte A, Galasko D, Gauthier S, Jicha G, et al: Research criteria for the diagnosis of Alzheimer's disease: Revising the NINCDS-ADRDA criteria. Lancet Neurol 6: 734-746, 2007.

13. Hallgren B and Sourander P: The effect of age on the non-haemin iron in the human brain. J Neurochem 3: 41-51, 1958.

14. Lan AP, Chen J, Chai ZF and Hu Y: The neurotoxicity of iron, copper and cobalt in Parkinson's disease through ROS-mediated mechanisms. Biometals 29: 665-678, 2016.

15. Wu Y, Passananti M, Brigante M, Dong W and Mailhot G: Fe(III)-EDDS complex in Fenton and photo-Fenton processes: From the radical formation to the degradation of a target compound. Environ Sci Pollut Res Int 21: 12154-12162, 2014.

16. Connor JR, Menzies SL, St Martin SM and Mufson EJ: A histochemical study of iron, transferrin, and ferritin in Alzheimer's diseased brains. J Neurosci Res 31: 75-83, 1992.

17. Grundke-Iqbal I, Fleming J, Tung YC, Lassmann H, Iqbal K and Joshi JG: Ferritin is a component of the neuritic (senile) plaque in Alzheimer dementia. Acta Neuropathol 81: 105-110, 1990.

18. Liu B, Moloney A, Meehan S, Morris K, Thomas SE, Serpell LC, Hider R, Marciniak SJ, Lomas DA and Crowther DC: Iron promotes the toxicity of amyloid beta peptide by impeding its ordered aggregation. J Biol Chem 286: 4248-4256, 2011. 
19. Egaña JT, Zambrano C, Nuñez MT, Gonzalez-Billault C and Maccioni RB: Iron-induced oxidative stress modify tau phosphorylation patterns in hippocampal cell cultures. Biometals 16: 215-223, 2003.

20. Ayton S, Faux NG and Bush AI: Association of cerebrospinal fluid ferritin level with preclinical cognitive decline in APOE- $\varepsilon 4$ carriers. JAMA Neurol 74: 122-125, 2017.

21. Haacke EM, Makki M, Ge Y, Maheshwari M, Sehgal V, $\mathrm{Hu}$ J, Selvan M, Wu Z, Latif Z, Xuan Y, et al: Characterizing iron deposition in multiple sclerosis lesions using susceptibility weighted imaging. J Magn Reson Imaging 29: 537-544, 2009.

22. Schiepers OJ, van Boxtel MP, de Groot RH, Jolles J, de Kort WL, Swinkels DW, Kok FJ, Verhoef P and Durga J: Serum iron parameters, HFE C282Y genotype, and cognitive performance in older adults: Results from the FACIT study. J Gerontol A Biol Med Sci 65: 1312-1321, 2010.
23. Milward EA, Bruce DG, Knuiman MW, Divitini ML, Cole M, Inderjeeth CA, Clarnette RM, Maier G, Jablensky A and Olynyk JK: A cross-sectional community study of serum iron measures and cognitive status in older adults. J Alzheimers Dis 20: 617-623, 2010.

24. Wang ZX, Tan L, Wang HF, Ma J, Liu J, Tan MS, Sun JH, Zhu XC, Jiang T and Yu JT: Serum iron, zinc, and copper levels in patients with Alzheimer's disease: A replication study and meta-analyses. J Alzheimers Dis 47: 565-581, 2015.

25. House MJ, St Pierre TG, Milward EA, Bruce DG and Olynyk JK: Relationship between brain $\mathrm{R}(2)$ and liver and serum iron concentrations in elder men. Magn Reson Med 63: 275-281, 2010.

26. Lin D, Ding J, Liu JY, He YF, Dai Z, Chen CZ, Cheng WZ, Zhou J and Wang X: Decreased serum hepcidin concentration correlates with brain iron deposition in patients with HBV-related cirrhosis. PLoS One 8: e65551, 2013. 